\title{
Removal of sulfamethoxazole and sulfapyridine by carbon nanotubes in fixed-bed columns
}

\author{
Yuan Tian, Bin Gao, Verónica L. Morales, Hao Chen, Yu Wang, and Hui Li
}

NOTICE: this is the author's version of a work that was accepted for publication in Chemosphere. Changes resulting from the publishing process, such as peer review, editing, corrections, structural formatting, and other quality control mechanisms may not be reflected in this document. Changes may have been made to this work since it was submitted for publication. A definitive version was subsequently published in Chemosphere, [Vol. 90, Issue 10, (2013)] DOI http://dx.doi.org/10.1016/j.chemosphere.2012.11.010 


\section{Removal of sulfamethoxazole and sulfapyridine by carbon nanotubes in fixed-bed columns}

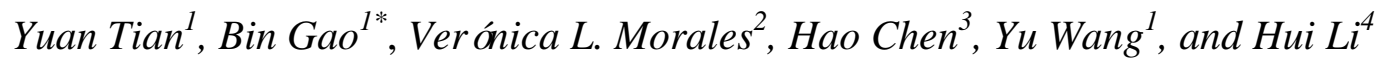

1. Department of Agricultural and Biological Engineering, University of Florida, Gainesville, FL 32611

2. SIMBIOS Centre, University of Abertay, Dundee, DDI U.K

3. Department of Soil and Water Science, University of Florida, Gainesville, FL 32611

4. Department of Crop and Soil Sciences, Michigan State University, East Lansing, MI 48824

*Corresponding Author, Phone: (352) 392-1864 ext.285, email: bg55@ufl.edu 


\begin{abstract}
Sulfamethoxazole (SMX) and sulfapyridine (SPY), two representative sulfonamide antibiotics, have gained increasing attention because of the ecological risks these substances pose to plants, animals, and humans. This work systematically investigated the removal of SMX and SPY by carbon nanotubes (CNTs) in fixed-bed columns under a broad range of conditions including: CNT incorporation method, solution $\mathrm{pH}$, bed depth, adsorbent dosage, adsorbate initial concentration, and flow rate. Fixed-bed experiments showed that $\mathrm{pH}$ is a key factor that affects the adsorption capacity of antibiotics to CNTs. The Bed Depth Service Time model describes well the relationship between service time and bed depth and can be used to design appropriate column parameters. During fixedbed regeneration, small amounts of SMX (3\%) and SPY (9\%) were irreversibly bonded to the $\mathrm{CNT} /$ sand porous media, thus reducing the column capacity for subsequent reuse from 67.9 to $50.4 \mathrm{mg} / \mathrm{g}$ for SMX and from 91.9 to $72.9 \mathrm{mg} / \mathrm{g}$ for SPY. The reduced column capacity resulted from the decrease in available adsorption sites and resulting repulsion (i.e., blocking) of incoming antibiotics from those previously adsorbed. Findings from this study demonstrate that fixed-bed columns packed with CNTs can be efficiently used and regenerated to remove antibiotics from water.

Keywords: antibiotics, nanotechnology, water treatment, filtration, removal efficiency
\end{abstract}




\section{Introduction}

Sulfonamide antibiotics are widely used as human and veterinary pharmaceuticals (Halling-Sørensen et al., 1998; Kolpin et al., 2002; Thiele-Bruhn, 2003). Sulfamethoxazole (SMX) and sulfapyridine (SPY) are two commonly used sulfonamide antibiotics primarily used for treating human patients, and are well known to bioaccumulate up the food chain and trigger acute as well as chronic adverse effects. (Göbel et al., 2005). Recent findings indicate that simultaneous exposure to multiple antibiotics could result in the enhanced toxic effects (Park and Choi, 2008). A general concern for public health is the development of antibiotic resistance from chronic exposure to antibiotic contaminated water. Therefore, it is urgent to investigate the pathways through which SMX and SPY disperse in the environment, and develop systems that can efficiently remove these dissolved substances from water.

Entry pathways of sulfonamide antibiotics to aquatic environments include aquaculture activity, pharmaceutical manufacturing, and medical waste disposal. Additionally, access could be indirectly gained to surface and subsurface waters from leached waste of livestock receiving antibiotic treatment (Baran et al., 2011). Precipitation events could further accelerate the release of antibiotics concentrated in animal manure, which, once dissolved in surface waters, could mix and load groundwater within the soil profile. The protection of surface and groundwater quality, as two primary sources for drinking water, from contamination of leached antibiotics is of great priority for pubic and environmental health.

A variety of physiochemical techniques have been developed to remove or destroy antibiotics from water sources, including oxidation (Beltrán et al., 2008), ion exchange 
(Choi et al., 2007), reverse osmosis (Adams et al., 2002), and adsorption (Ternes et al., 2002). Adsorption is a standard method used to remove dissolved contaminants from water. Adsorbents, such as clay (Avisar et al., 2010), zeolite (Braschi et al., 2010), and activated carbon (Caliskan and Gokturk, 2010), have been examined for their removal efficiency of sulfonamide antibiotics in aqueous solutions. Solution chemistry has been shown to strongly affect the removal efficiency of these adsorbents with particular importance placed on solution pH (Lertpaitoonpan et al., 2009), ionic strength (Gao and Pedersen, 2005), and presence of competitive sulfonamide antibiotics (Gao and Pedersen, 2010).

Carbon nanotubes (CNTs), as novel sorbents, have gained increasing attention because of their exceptional sorbing properties. CNTs have hollow and layered structures with a characteristically large surface area, thus endowing CNTs with great potential for superior sorption capability (Wang et al., 2009). Previous studies have demonstrated high adsorption ability of CNTs to both heavy metals (Stafiej and Pyrzynska, 2007) and organic pollutants (Zhang et al., 2010). Investigations on the adsorption of sulfonamide antibiotics by CNTs have reported that removal efficiency varies according to the quantity of walls making up the CNTs as well as to chemical pre-treatment of the CNTs (Ji et al., 2009; Ji et al., 2010). Pretreatment of CNTs (e.g., by surface functionalization) is widely used to improve the dispersion of these nanoparticles in aqueous solutions (Tian et al., 2011). The introduction of functional groups also increases the ion-exchange capacity of the CNTs, thereby augmenting the number of available sites that can participate in electrostatic adsorption (Atieh et al., 2010). As such, functionalized CNTs 
have been reported to exhibit greater potential for removing antibiotics from aqueous environments than pristine CNTs (Zhang et al., 2010).

The current application of CNTs for antibiotic removal from water has been restricted to batch sorption methods. Two key disadvantages of batch methods include difficulty to collect exhausted/spent adsorbents and interruptions incurred when integrated to existing continuous processes (Eckenfelder, 2000). For example, the in-situ removal of antibiotics in the environment (e.g., an agricultural field) requires a continuous flow setup to cope with the intermittent discharge of antibiotic-loaded runoff. It is thus necessary to develop and optimize CNT-enabled water treatment methods that take advantage of the large sorption capacity of CNTs in a setup that can be deployed in the field.

Unlike batch sorption systems, fixed-bed filters permit continuous flow and adsorption of antibiotics from solution; thus, opening a wealth of opportunities for in situ water treatment. The fixed-bed filters enabled by high performance adsorbents exploit the high sorption capacity of the adsorbent, while enabling the practicalities of continuous flow operation. Despite the above-mentioned benefits of fixed-bed systems and great sorption potential of CNTs (Tian et al., 2012b), to the authors' knowledge, the removal of antibiotics from aquatic environment using CNT-enabled fixed-bed columns has not been explored.

In this work, we used CNT-enabled fixed-bed column methods to investigate the removal of SMX and SPY from aqueous solutions under various conditions. Our overarching objective was to investigate the removal efficiency of SMX and SPY by CNTs in a fixed-bed system under various physicochemical conditions. The specific 
objectives of the work were to (1) examine the removal of SMX and SPY from aqueous solutions by trickling antibiotic-contaminated water through a CNT/sand fixed-bed column under conditions of varying $\mathrm{pH}, \mathrm{CNT}$ incorporation method, adsorbent dosage, bed depth, adsorbate initial concentration, and flow rate, and (2) evaluate the efficiency of regeneration of the fixed-bed columns for reuse.

\section{Materials and methods}

\subsection{Bed materials and conditions}

Functionalized multi-walled carbon nanotubes (CNTs) (Cheap Tubes Inc., Brattleboro, VT) and quartz sand were used as filter materials in the fix-bed columns. Three methods of CNT incorporation to the sandy medium were tested (layered, mixed, and deposited), bed depths tested ranged from 6 to $15 \mathrm{~cm}$, and flow rates ranged from 1 to $2 \mathrm{~mL} \min ^{-1}$. Chemical conditions tested for fixed-bed experiments were 3.0 to $9.0 \mathrm{pH}, 10$ to $40 \mathrm{mg}$ adsorbent dosage, and 10 to $40 \mathrm{mg} \mathrm{L}^{-1}$ of antibiotic concentration.

CNTs were produced using a chemical vapor deposition method with nickel and magnesium catalysts. Subsequent functionalization was achieved with an acid mixture of concentrated sulfuric and nitric acids $(3: 1, \mathrm{v}: \mathrm{v})$ to introduce carboxyl and hydroxyl functional groups to the nanotube surface (Zhang et al., 2011). A batch of the functionalized CNTs were used in the dry powder form (undispersed CNTs), while a second batch was dispersed in water to create a suspension of CNTs (dispersed CNTs). To make the dispersion, $16 \mathrm{mg}$ of the synthesized CNT powder were dispersed in 1000 $\mathrm{ml}$ deionized water and subsequently sonicated for 30 minutes in a Misonix S3000 ultrasonicator (QSonica, Newtown, CT). 
Thorough characterization of the CNTs for physiochemical properties was performed for the following properties. Surface area of undispersed CNTs was measured using the NOVA 1200 surface area analyzer (Quantachrome Instruments, Boynton Beach, FL), following the Brunauer-Emmett-Teller (BET) nitrogen adsorption method at $77 \mathrm{~K}$. Point of zero charge (PZC) of the undispersed CNTs was determined using the mass titration method (Noh and Schwarz, 1990). Hydrodynamic diameter of dispersed CNTs was determined by dynamic light scattering with a Brookhaven ZetaPlus (Brookhaven Instruments Corporation, Holtsville, NY). CNT concentration in suspension was calibrated by measuring the total absorption of light at wavelengths of $255 \mathrm{~nm}$ using Evolution 60 UV-Vis Spectrophotometer (Thermo Scientific, Waltham, MA).

Quartz sand (Standard Sand \& Silica Co., Davenport, FL) was sieved into 0.5 - 0.6 $\mathrm{mm}$ grain size and washed using deionized water (DI water). Basic properties and surface elemental compositions of the sand were reported previously by the authors (Tian et al., 2012a).

\subsection{Antibiotics}

Two sulfonamide antibiotics, sulfamethoxazole (SMX) and sulfapyridine (SPY) (99\%, Sigma-Aldrich Co., St. Louis, MO), were used as adsorbates in the fixed-bed column experiments. Chemical structures and properties of SMX and SPY are reported in Table S1 (Supporting Information). Stock solutions of SMX/SPY were prepared (200 $\mathrm{mg} / \mathrm{L}$ ) and subsequently spiked with either $0.1 \mathrm{M} \mathrm{KOH}$ or $\mathrm{HCl}$ to adjust for desired $\mathrm{pH}$ $(3.0-9.0)$ of the final solution. Antibiotic concentrations were determined by light absorption at wavelengths of $265 \mathrm{~nm}$ with a UV-Vis Spectrophotometer (Caliskan and 
Gokturk, 2010). Lack of temporal change of concentration of SMX and SPY confirmed their stability for the duration of our experiments (data not shown).

\subsection{Fixed-bed column experiments}

Fixed-bed column experiments were used to investigate the removal of dissolved antibiotics from water in a CNT/sand fixed-bed system. A summary of the conditions tested is presented in Table 1, which includes CNT incorporation methods, $\mathrm{pH}$, bed depth, adsorbent dosage, and adsorbate initial concentration, and flow rate.

An acrylic column of $2.5 \mathrm{~cm}$ in diameter and $15 \mathrm{~cm}$ height was used to contain the $\mathrm{CNT} /$ sand system, to which appropriate amount of CNTs (Table 1) were packed with $146.8 \mathrm{~g}$ of sand for all packing methods. Wet-packing of the porous medium was used in order to minimize layering of and air entrapment in the porous medium. The porosity of all the CNT/sand columns was approximately 0.40 . A peristaltic pump was connected at the top of the column (inlet) to regulate the flow rate. Additionally, membranes with 50 $\mu \mathrm{m}$ pores (Spectra/Mesh, Spectrum Laboratories, Inc.) were used at the inlet and outlet of the column to distribute the flow over the cross-sectional area of the fixed-bed.

For the tested nanoparticle incorporation methods, CNTs were added to the sand medium by means of layering, mixing, or deposition (Tian et al., 2012b). For the CNT layered method, undispersed CNTs were incorporated to the system as a layer on top of the wet-packed sand column. For the CNT mixed method, undispersed CNTs were first mixed with sand and subsequently wet-packed into the column. For the CNT deposited method, the column was first wet-packed sand and a dispersion of CNTs injected through the column with a peristaltic pump (Masterflex L/S, Cole Parmer Instrument, Vernon 
Hills, IL) in a downward flow direction for more than $10 \mathrm{~h}$. Then, the influent was changed to DI water was for $2 \mathrm{~h}$ to elute suspended (not deposited) CNTs from the column. Effluent samples were collected at the outlet of the column with a fraction collector (IS-95 Interval Sampler, Spectrum Chromatography, Houston, TX) to monitor the concentration of eluted CNTs. Dispersed CNT concentration was determined by total absorption of light at wavelengths of $255 \mathrm{~nm}$ with UV-Vis Spectrophotometer. Mass balance calculations of injected and eluted CNTs were used to determine the amount of retained CNTs in the column. It is important to note that prior to use for antibiotic sorption experiments, no CNTs were detected in the effluent of the CNT/sand packed columns; therefore, CNT incorporation to the sandy medium considered irreversible. A column packed only with natural sand was used as the control.

After packing the column with the methods described above, an antibiotic-free background solution was injected to the column for $2 \mathrm{hr}$ until the pore water chemistry reached equilibrium. Subsequently, an antibiotic solution was continuously injected to column and effluent samples collected at fixed intervals with a fraction collector. The concentration of antibiotics in the column effluent was then measured with UV-Vis Spectrophotometer and breakthrough curves built with this information. The experiment was terminated when the effluent concentration matched the initial concentration. All the column sorption studies were performed in duplicate.

\subsection{Column regeneration and recycling}

Spent fixed-bed columns (i.e., as resulted from antibiotic sorption tests described in the section prior) were used to evaluate bed regeneration and recycling efficiency. 
Experimental conditions of the selected columns included: CNT incorporation by mixing, $\mathrm{pH}$ of 5.6, adsorbent dosage of $10 \mathrm{mg}$, bed depth of $15 \mathrm{~cm}$, adsorbate initial concentration of $20 \mathrm{mg} / \mathrm{L}$, and flow rate of $2 \mathrm{ml} / \mathrm{min}$.

To regenerate the spent columns, a solution containing $30 \mathrm{~g} / \mathrm{L} \mathrm{NaCl}$ and $1.5 \mathrm{~g} / \mathrm{L}$ $\mathrm{NaOH}$ of $\mathrm{pH} 12$ was injected to the fixed-bed columns at the same flow rate as that used for antibiotic sorption experiments until effluent SMX or SPY concentrations fell below the detection limit (Faki et al., 2008). Effluent samples were collected and analyzed using the same protocol as that in the column filtration experiment. The column recycling was used to evaluate the viability of reuse of the CNT/sand fixed-bed column. After regeneration of the column and equilibration with background solution, column adsorption and sample analysis were performed under the same conditions. The column was recycled up to five times and column capacity at each reuse cycle determined. All the column regeneration and recycling studies were performed in duplicate.

\section{Results and discussion}

\subsection{Column analyses}

The two antibiotics showed very high mobility in the CNT-free fixed-bed filters (Figure 1), which can be attributed to their low reactivity with the sand media (Chen et al., 2011). Columns enabled with CNTs, however, showed high retention ability for both SMY and SPY (Figure 1), indicating that CNTs can be used in fixed-bed columns to remove antibiotics in solution. For all tested conditions, the CNT/sand fixed-bed column capacity ranged from $11.7-92.0 \mathrm{mg} / \mathrm{g}$ for SMX and $77.8-123.4 \mathrm{mg} / \mathrm{g}$ for SPY (Table 1). 
The primary adsorption zone (PAZ) concept developed by Gupta et al (Gupta et al., 1997; Gupta et al., 2000) was used to analyze the breakthrough data of the antibiotics in the CNT/sand columns. During antibiotics injection into the fixed-bed column, the PAZ initially formed as a narrow band at the top of column. As column operation continues, the upper layers of the adsorbents (CNTs) become saturated with incoming antibiotics and the PAZ advances downward through the fixed-bed column until it reaches the bottom most layer of the column (Kundu and Gupta, 2005; Sousa et al., 2010). At this point, the injected antibiotics begin to breakthrough the column, as detectable in the effluent samples. In this work, normalized concentrations $(\mathrm{C} / \mathrm{Co})$ of antibiotics in the effluent were plotted against the operation time (min) to obtain the breakthrough curves of the antibiotics in the columns.

Parameters controlling the formation and movement of the PAZ can be quantitatively determined form the breakthrough data (Benefield et al., 1982; Kundu and Gupta, 2005). Definition of the PAZ parameters (e.g., $t_{b}, t_{e}, v_{z}$ et al.) used in this study is provided in Table 2 . These parameters were calculated to quantitatively assess the effect of $\mathrm{pH}(3.0,5.6,7.0$, or 9.0), CNT incorporation method (layered, mixed, or deposited), adsorbent dosage $(10,20,30$, or $40 \mathrm{mg})$, bed depth $(6,9,12$, or $15 \mathrm{~cm})$, adsorbate initial concentration $\left(10,20,30\right.$, or $\left.40 \mathrm{mg} \mathrm{L}^{-1}\right)$, and flow rate $\left(1,2,3\right.$, or $\left.4 \mathrm{~mL} \mathrm{~min}^{-1}\right)$, on the removal efficiency of CNT/sand columns to the two antibiotics. The determined PAZ and column adsorption parameters for all the experimental conditions tested are summarized in Table 1 and details provided in the supporting information (Tables S2-S7). 


\subsection{Effect of CNT incorporation method}

The effect of CNT incorporation method on the adsorption and transport of SMX and SPY in the columns is illustrated in Figure 1. The column maximum adsorption capacities to SMX and SPY, defined as mg of antibiotic per $\mathrm{g}$ of CNTs, are summarized in Table 1. Experimental results indicate that the column adsorption capacity for SMX was $68.5 \mathrm{mg} / \mathrm{g}$ for layered method, $69.3 \mathrm{mg} / \mathrm{g}$ for mixed method, and $77.7 \mathrm{mg} / \mathrm{g}$ for dispersed method, with the capacity trend in the order of layered $<$ mixed $<$ dispersed. A similar performance in the CNT incorporation method was observed for experiments filtering SPY antibiotic. Likewise, the time to column exhaustion, $t_{e}$, (as reported in Table 1) indicates that CNT incorporation by the dispersed method prolongs antibiotic sorption time the most; thus, increasing the column's sorption capacity. As presented in the supporting information (Table S2), the time required for the PAZ to form, $t_{f}$, and time required for the PAZ to move through the fixed-bed, $t_{z}$, followed the same trend for the tested CNT incorporation methods. However, from a practical perspective the construction of a CNT-enabled fixed-bed column by the mixed CNT method is recommended with little compromise on the sorption performance of the system. Therefore, the remaining facets of the study are focused on fixed-bed systems built with the mixed CNT method.

\subsection{Effect of $\mathrm{pH}$}

The effect of solution $\mathrm{pH}$ on the adsorption and transport of SMX and SPY in the columns is illustrated in Figure 2. Experimental results showed that the breakpoint time , $t_{b}$, decreased from 33.4 to 16.6 min with the increase of $\mathrm{pH}$ from 3.0 to 9.0 (Table 1 ), 
indicating that $\mathrm{pH}$ induced accelerated transport of SMX and SPY in the columns as conditions became more alkaline. As presented in the supporting information (Table S3), the inverse correlation between $t_{f}$ and $\mathrm{pH}$ indicates that the $\mathrm{PAZ}$ formed faster at higher $\mathrm{pH}$ conditions. Similarly, shorter $t_{z}$ and faster $v_{z}$ with higher $\mathrm{pH}$ conditions indicate that PAZ forms and moves faster under these conditions. Table 1 shows that, when $\mathrm{pH}$ was increased from 3.0 to 9.0, the column adsorption capacity of SMX and SPY decreased from 84.9 to $11.7 \mathrm{mg} / \mathrm{g}$ and 93.9 to $77.8 \mathrm{mg} / \mathrm{g}$, respectively. This suggests that alkaline $\mathrm{pH}$ dramatically decrease of the column ability to remove antibiotics, especially for SMX.

Mass titration measurements indicated that the PZC of CNTs was 2.2, signifying that the surface of CNT was negatively charged for all the treatments tested in the present study. The surface charge of SMX and SPY varied greatly with $\mathrm{pH}$, as represented by the acidity constants $\left(\mathrm{pK}_{\mathrm{a} 1}=1.8, \mathrm{pK}_{\mathrm{a} 2}=5.6\right.$ for $\mathrm{SMX}$ and $\mathrm{pK}_{\mathrm{a} 1}=2.3, \mathrm{pK}_{\mathrm{a} 2}=8.4$ for SPY). Table S1 in the Supporting Information provides the details of the speciation of SMX and SPY at the four solution $\mathrm{pH}$ values used this study. SMX and SPY became increasingly negatively charged with increasing solution $\mathrm{pH}$; thus promoting electrostatic repulsion between CNTs and antibiotics. The larger fractions of neutral antibiotic species for SPY relative to those of SMX at the four $\mathrm{pH}$ levels tested explain well the observed differences in sorption capacity, by way of $\mathrm{pH}$ dependent speciation and its respective electrostatic phenomenon. For example, adsorption capacity at $\mathrm{pH} 7$ for SPY was recorded at $86.1 \mathrm{mg} \mathrm{g}^{-1}$, while that for SMX was $45.8 \mathrm{mg} \mathrm{g}^{-1}$. The antibiotic speciation at the same $\mathrm{pH}$ was dominated by $\mathrm{SMX}^{-}(96 \%)$ or neutral SPY (96\%), which is expected to exhibit different degrees of electrostatic repulsion when interacting with CNT surfaces. In 
agreement with the antibiotic speciation effect, Figure 2 illustrates the large effect of $\mathrm{pH}$ on adsorption capacity of SMX when passing through the CNT/sand fixed-bed column, while a negligible effect is observed on adsorption capacity of SPY for identical experiments.

Beside the electrostatic phenomena described above, $\pi-\pi$ interactions between functional groups are suspected to contribute to the adsorption of SMX and SPY by CNTs, as has been previously been implicated for sorption of phenolic compounds by CNTs (Lin and Xing, 2008). Amine functional groups and N-heteroaromatic rings in SMX and SPY are known to serve as $\pi$-acceptors; whereas, carboxyl and hydroxyl functional groups on benzene rings in CNTs can serve as both $\pi$-acceptors and $\pi$-donors (Zhang et al., 2010). Thus the authors propose that the attraction of acceptor-acceptor and acceptor-donor pairs between the antibiotics and the CNTs is an additional mechanism responsible for the observed strong adsorption of antibiotics onto CNTs at lower $\mathrm{pH}$ values.

\subsection{Effect of bed depth}

The effect of bed depth on the adsorption and transport of SMX and SPY in the columns is illustrated in Figure 3. The column adsorption capacities at various bed depths were in general greater for SPY (91.9 to $97.1 \mathrm{mg} \mathrm{g}^{-1}$ ) than for SMX (69.3 to $72.8 \mathrm{mg} \mathrm{g}^{-1}$ ) (Table 1).

To evaluate the effect of bed depth on the breakthrough time, the breakthrough data were interpreted using the Bed Depth Service Time (BDST) model. The BDST model was first introduced by (Bohart and Adams, 1920) and further developed by (Hutchins, 
1973). This model has primarily been used in the analysis of fixed-bed breakthrough data of heavy metals (Ko et al., 2000; Cortes-Martinez et al., 2009) and organic pollutants (Abu-Lail et al., 2010; Patel and Vashi, 2012). The relationship between service time and bed depth is as follows:

$$
t=\frac{N_{0}}{C_{0} v} h-\frac{1}{k C_{0}} \ln \left(\frac{C_{0}}{C_{t}}-1\right)
$$

where $t$ is the service time at breakthrough [min], $N_{0}$ is the dynamic adsorption capacity of the bed $\left[\mathrm{mg} \mathrm{L}^{-1}\right], C_{0}$ is the influent antibiotics concentration $\left[\mathrm{mg} \mathrm{L}^{-1}\right], v$ is the linear flow velocity of feed to the fixed-bed [cm $\left.\min ^{-1}\right], h$ is the CNT/sand bed depth [cm], $k$ is the adsorption rate constant $\left[\mathrm{L} \mathrm{mg}^{-1} \mathrm{~min}^{-1}\right]$, and $C_{t}$ is the effluent solute concentration at time $t\left[\mathrm{mg} \mathrm{L}^{-1}\right]$.

The linear relationship between $t$ and $h$ (Figure 4) was described by the slope $\frac{N_{o}}{C_{o}}$ and the intercept $\frac{1}{k C_{0}} \ln \left(\frac{C_{0}}{C_{t}}-1\right)$. The values of $N_{0}$ and $k$ were thereafter back calculated. The critical bed depth, $h_{0}$, is the minimum depths of CNT/sand required to achieve the effluent breakthrough, which can be calculated from the lines best fitting equations by letting $\mathrm{t}=0$ (Kundu and Gupta, 2005; Mohan and Sreelakshmi, 2008).

The BDST model was developed for breakpoint, $t_{b}$, and exhausted point, $t_{e}$, respectively. Plots of bed depth against service time are presented in the Figure 4 with their respective linear regression. The excellent regression results $\left(\mathrm{R}^{2}>0.9\right)$ permit the fitted curves to be used for predicting the service time corresponding to a given bed depth both for SMX or SPY. Moreover, the identified relationship between bed depth and service time points out that changes in $t_{b}$ are paralleled by changes in $t_{e}$; thus indicating 
that the time required for the PAZ to move the length of its own height, $t_{z}$, is independent of bed depth. The horizontal distance between the two parallel lines of Figure 4 is taken as the height of the exchange zone (Kundu and Gupta, 2005), resulting in heights of 4.5 and $5.2 \mathrm{~cm}$ for SMX and SPY, respectively.

The additional BDST parameters $N_{0}, k$ and $h_{0}$ were then calculated using the estimated regression values for breakpoint at different bed depths. Values of $N_{0}, k$, and $h_{0}$ for SMX were estimated at $16.58 \mathrm{mg} \mathrm{L}^{-1}, 0.038 \mathrm{~L} \mathrm{mg}^{-1} \mathrm{~min}^{-1}$, and $1.81 \mathrm{~cm}$; while for SPY estimated values were of $20.72 \mathrm{mg} \mathrm{L}^{-1}, 0.023 \mathrm{~L} \mathrm{mg}^{-1} \mathrm{~min}^{-1}$, and $2.42 \mathrm{~cm}$, These BDST parameters suggest that the removal ability of CNTs for SPY is better than that for SMX under the same conditions. For up-scaling of CNT/sand fixed-bed systems, the BDST model could be used to optimize the design and performance of large-scale fixed-bed columns for the removal of antibiotics from water (Mohan and Sreelakshmi, 2008).

\subsection{Effect of adsorbent dosage}

The effect of adsorbent dosage on the adsorption and transport of SMX and SPY in the columns is illustrated in Figure S1 (Supporting Information). As expected, the breakpoint time, $t_{b}$, increased with the increase in CNT dosage. As reported in Table 1, a four-fold increase of CNT in the column ensued an increase in $t_{b}$ from 27.5 to $66.5 \mathrm{~min}$ for SMX and 33.5 to $85.5 \mathrm{~min}$ for SPY. Conversely, the column adsorption capacity decreased from 69.3 to $62.8 \mathrm{mg} / \mathrm{g}$ for SMX and 91.9 to $82.4 \mathrm{mg} / \mathrm{g}$ for SPY for the same increase in CNT dosage. From a fixed-bed capacity standpoint, it is clear that the mass of adsorbed antibiotics per unit mass of CNTs decreased with greater amount of CNTs in the fixed-bed, thus reducing the performance efficiency of the fixed-bed filter. A possible 
explanation for the decrease in fixed-bed efficiency could be attributed to $\pi$-bond interference of the additional CNTs with each other; thus preventing the formation of $\pi$ bonds between antibiotics and the CNTs in the column (Ozdemir et al., 2009; MontazerRahmati et al., 2011).

\subsection{Effect of adsorbate initial concentration}

The effect of adsorbate initial concentration on the adsorption and transport of SMX and SPY in the columns is illustrated in Figure S2 (Supporting Information). As shown in the figure, the breakpoint time was shortest with the highest SMX and SPY initial concentration, and increased as initial concentration decreased. The breakpoint time decreased from 37.5 to $25.5 \mathrm{~min}$ for SMX and 51.5 to $25.5 \mathrm{~min}$ for SPY as the initial concentration of antibiotics quadrupled. The column adsorption capacity of antibiotics by CNT was increased from 54.8 to $92.0 \mathrm{mg} \mathrm{g}^{-1}$ for SMX and 80.9 to $123.4 \mathrm{mg} \mathrm{g}^{-1}$ for SPY for the same increase in antibiotic initial concentration (Table 1). This large increase in fixed-bed capacity is ascribed to the larger concentration gradient generated that promotes greater mass transfer of antibiotics from the liquid phase to the solid phase (i.e., the CNT surface) (Nasuha et al., 2010).

\subsection{Effect of flow rate}

The effect of flow rate on the adsorption and transport of SMX and SPY in the columns is illustrated in Figure S3 (Supporting Information). As shown in the figure, the breakpoint time decreased with increasing flow rates from 59.1 to 14.8 min for SMX and 67.1 to $16.8 \mathrm{~min}$ for SPY (Table 1). The column removal capacity of antibiotics by CNT 
was decreased from 78.3 to $61.9 \mathrm{mg} \mathrm{g}^{-1}$ for SMX and 94.9 to $83.6 \mathrm{mg} \mathrm{g}^{-1}$ for SPY when flow rate was increased from 1 to $4 \mathrm{ml} \mathrm{min}^{-1}$. This reduction in sorption capacity is likely due to the decrease in contact time between the antibiotics and CNTs at higher flow rates. The increase of flow rate also accelerates the movement of PAZ downwards in the fixedbed, which contributes to decreased adsorption capacity and column efficiency (Ozdemir et al., 2009).

\subsection{Column regeneration and recycling}

The release curves of SMX and SPY in the post-adsorption columns during the regeneration process are presented in the supporting information (Figure S4). Results showed that the release of SMX and SPY produced a maximum effluent concentration of $1.2 \mathrm{C} / \mathrm{C}_{0}$ after $22 \mathrm{~min}$ of regeneration treatment. The mass recovery for the regeneration process was $97 \%$ for SMX and $91 \%$ for SPY, suggesting that $3 \%$ of SMX and $9 \%$ of SPY of the previously sorbed antibiotics were strongly and irreversibly bonded to the $\mathrm{CNT} /$ sand porous media at each regeneration cycle. Thus, although the sorption capacity of the fixed-beds diminished after each adsorption-regeneration cycle, the fixed-beds continued to have an acceptable capacity to remove antibiotics from contaminated waters.

The change of column capacity for 5 consecutive regeneration cycles for each antibiotic are presented in Figure 5. From these plots it is evident that column capacity decreased after reuse from 67.9 to $32.6 \mathrm{mg} \mathrm{g}^{-1}$ for SMX and from 91.9 to $40.0 \mathrm{mg} \mathrm{g}^{-1}$ for SPY. As suggested previously, the reduced column capacity could be a result of decreased available adsorption sites (i.e., blocking) and repulsion by the irreversibly sorbed antibiotics from previous cycles. It is important to note that although the absolute 
sorption capacity of the CNT-sand fixed-beds is lower for SMX than for SPY on a mg g ${ }^{-1}$ basis, regeneration of the columns after 5 cycles reduced the sorption capacity of both antibiotics by a similar fraction (52\% for SMX and $56 \%$ for SPY).

\section{Conclusions}

The removal of sulfamethoxazole (SMX) and sulfapyridine (SPY) from aqueous solutions by fixed-bed columns was tested under various conditions. Experimental results indicate that, from the multiple factors that can be varied in a fixed-bed filtration system (CNT incorporation, $\mathrm{pH}$, bed depth, adsorbent dosage, adsorbate initial concentration, and flow rate), the effect of $\mathrm{pH}$ affects column adsorption capacity most strongly. The mechanism driving this phenomenon were attributed to the speciation of negative, neutral and positive antibiotics molecules at the tested $\mathrm{pH}$ levels, the level of protonation the functional groups on the CNT surfaces, and on possible $\pi$-bonds between the adsorbant and adsorbate. Of the three CNT incorporation methods tested, columns built with dispersed CNTs rendered the columns with a greater adsorption capacity than those built with undispersed CNTs due to the greater CNT surface area within the column. The Bed Depth Service Time (BDST) model describes well the relationship between service time and bed depth and further permits estimation of optimum column parameters. The increase of adsorbent dosage slightly decreases the capacity of the filters while extending the lifetime of the filter, but penalizes fixed-bed efficiency. Higher adsorbate initial concentration offers greater mass transfer driving force to move antibiotics in the liquid phase towards the CNTs surface; thus, enhancing the efficiency of antibiotic sorption. The increase of flow rate results in the decrease of contact time between the antibiotics 
and CNTs. This accelerates the movement of PAZ downwards in the fixed-bed prior to becoming saturated, thus contributing to the overall decrease in adsorption capacity. Regeneration of the fixed-beds was successfully achieved, demonstrating that only a small fraction of SMX and SPY were irreversibly sorbed onto the CNT/sand porous

medium. As a result, each regeneration cycle reduced the capacity of the bed by $8-26 \%$ for both types of antibiotics, but permitted the column to be reused multiple times with an acceptable antibiotic sorption capacity. With recent capacity scale-ups driving prices down (\$25-38 per kg) (Agboola et al., 2007), operational cost for CNTs in wastewater treatment could be lower than other commercial carbon sorbents, such as activated carbon (AC). It has been pointed out that CNTs are better adsorbents to antibiotics than some of the ACs under many circumstance (Pan and Xing, 2008).

\section{Acknowledgements}

This work was partially supported by the USDA through grant 2009-65102-05847 and the NSF through grant CBET-1054405. 


\section{References}

Abu-Lail, L., Bergendahl, J.A., Thompson, R.W., 2010. Adsorption of methyl tertiary butyl ether on granular zeolites: Batch and column studies. J Hazard Mater 178, 363-369.

Adams, C., Wang, Y., Loftin, K., Meyer, M., 2002. Removal of antibiotics from surface and distilled water in conventional water treatment processes. J Environ Eng 128, 253-260.

Agboola, A.E., Pike, R.W., Hertwig, T.A., Lou, H.H., 2007. Conceptual design of carbon nanotube processes. Clean Technol Environ Policy 9, 289-311.

Atieh, M.A., Bakather, O.Y., Al-Tawbini, B., Bukhari, A.A., Abuilaiwi, F.A., Fettouhi, M.B., 2010. Effect of carboxylic functional group functionalized on carbon nanotubes surface on the removal of lead from water. Bioinorg Chem Appl, 603978.

Avisar, D., Primor, O., Gozlan, I., Mamane, H., 2010. Sorption of sulfonamides and tetracyclines to montmorillonite clay. Water Air Soil Poll 209, 439-450.

Baran, W., Adamek, E., Ziemiańska, J., Sobczak, A., 2011. Effects of the presence of sulfonamides in the environment and their influence on human health. $\mathbf{J}$ Hazard Mater 196, 1-15.

Beltrán, F.J., Aguinaco, A., García-Araya, J.F., Oropesa, A., 2008. Ozone and photocatalytic processes to remove the antibiotic sulfamethoxazole from water. Water Res 42, 3799-3808.

Benefield, L.D., Judkins, J.F., Weand, B.L., 1982. Process chemistry for water and wastewater treatment. Prentice-Hall, Englewood Cliffs, N.J. 
Bohart, G.S., Adams, E.Q., 1920. Some aspects of the behavior of charcoal with respect to chlorine.1. J Am Chem Soc 42, 523-544.

Braschi, I., Blasioli, S., Gigli, L., Gessa, C.E., Alberti, A., Martucci, A., 2010. Removal of sulfonamide antibiotics from water: Evidence of adsorption into an organophilic zeolite y by its structural modifications. J Hazard Mater 178, 218-225.

Caliskan, E., Gokturk, S., 2010. Adsorption characteristics of sulfamethoxazole and metronidazole on activated carbon. Separ Sci Technol 45, 244-255.

Chen, H., Gao, B., Li, H., Ma, L.Q., 2011. Effects of ph and ionic strength on sulfamethoxazole and ciprofloxacin transport in saturated porous media. J Contam Hydrol 126, 29-36.

Choi, K.-J., Son, H.-J., Kim, S.-H., 2007. Ionic treatment for removal of sulfonamide and tetracycline classes of antibiotic. Sci Total Environ 387, 247-256.

Cortes-Martinez, R., Solache-Rios, M., Martinez-Miranda, V., Alfaro-Cuevas, R., 2009. Removal of cadmium by natural and surfactant-modified mexican zeolitic rocks in fixed bed columns. Water Air Soil Poll 196, 199-210.

Eckenfelder, W.W., 2000. Industrial water pollution control. McGraw-Hill, Boston, Mass. [u.a.].

Faki, A.e., Turan, M., Ozdemir, O., Turan, A.Z., 2008. Analysis of fixed-bed column adsorption of reactive yellow 176 onto surfactant-modified zeolite. Ind Eng Chem Res 47, 6999-7004.

Gao, J., Pedersen, J.A., 2010. Sorption of sulfonamide antimicrobial agents to humic acid-clay complexes. J Environ Qual 39, 228-235. 
Gao, J.A., Pedersen, J.A., 2005. Adsorption of sulfonamide antimicrobial agents to clay minerals. Environ Sci Technol 39, 9509-9516.

Göbel, A., Thomsen, A., McArdell, C.S., Joss, A., Giger, W., 2005. Occurrence and sorption behavior of sulfonamides, macrolides, and trimethoprim in activated sludge treatment. Environ Sci Technol 39, 3981-3989.

Gupta, V.K., Srivastava, S.K., Mohan, D., Sharma, S., 1997. Design parameters for fixed bed reactors of activated carbon developed from fertilizer waste for the removal of some heavy metal ions. Waste Manage 17, 517-522.

Gupta, V.K., Srivastava, S.K., Tyagi, R., 2000. Design parameters for the treatment of phenolic wastes by carbon columns (obtained from fertilizer waste material). Water Res 34, 1543-1550.

Halling-Sørensen, B., Nors Nielsen, S., Lanzky, P.F., Ingerslev, F., Holten Lützhøft, H.C., Jørgensen, S.E., 1998. Occurrence, fate and effects of pharmaceutical substances in the environment- a review. Chemosphere 36, 357-393.

Hutchins, R.A., 1973. New method simplifies design of activated-carbon systems. Chem Eng-new York 80, 133-138.

Ji, L.L., Chen, W., Zheng, S.R., Xu, Z.Y., Zhu, D.Q., 2009. Adsorption of sulfonamide antibiotics to multiwalled carbon nanotubes. Langmuir 25, 11608-11613.

Ji, L.L., Shao, Y., Xu, Z.Y., Zheng, S.R., Zhu, D.Q., 2010. Adsorption of monoaromatic compounds and pharmaceutical antibiotics on carbon nanotubes activated by koh etching. Environ Sci Technol 44, 6429-6436.

Ko, D.C.K., Porter, J.F., McKay, G., 2000. Optimised correlations for the fixedbed adsorption of metal ions on bone char. Chem Eng Sci 55, 5819-5829. 
Kolpin, D.W., Furlong, E.T., Meyer, M.T., Thurman, E.M., Zaugg, S.D., Barber, L.B., Buxton, H.T., 2002. Pharmaceuticals, hormones, and other organic wastewater contaminants in u.S. Streams, 1999-2000: A national reconnaissance. Environ Sci Technol 36, 1202-1211.

Kundu, S., Gupta, A.K., 2005. Analysis and modeling of fixed bed column operations on as(v) removal by adsorption onto iron oxide-coated cement (iocc). J Colloid Interf Sci 290, 52-60.

Lertpaitoonpan, W., Ong, S.K., Moorman, T.B., 2009. Effect of organic carbon and ph on soil sorption of sulfamethazine. Chemosphere 76, 558-564.

Lin, D., Xing, B., 2008. Adsorption of phenolic compounds by carbon nanotubes: Role of aromaticity and substitution of hydroxyl groups. Environ Sci Technol 42, 72547259.

Mohan, S., Sreelakshmi, G., 2008. Fixed bed column study for heavy metal removal using phosphate treated rice husk. J Hazard Mater 153, 75-82.

Montazer-Rahmati, M.M., Rabbani, P., Abdolali, A., Keshtkar, A.R., 2011. Kinetics and equilibrium studies on biosorption of cadmium, lead, and nickel ions from aqueous solutions by intact and chemically modified brown algae. J Hazard Mater 185, 401-407.

Nasuha, N., Hameed, B.H., Din, A.T.M., 2010. Rejected tea as a potential lowcost adsorbent for the removal of methylene blue. J Hazard Mater 175, 126-132.

Noh, J.S., Schwarz, J.A., 1990. Effect of hno3 treatment on the surface-acidity of activated carbons. Carbon 28, 675-682. 
Ozdemir, O., Turan, M., Turan, A.Z., Faki, A., Engin, A.B., 2009. Feasibility analysis of color removal from textile dyeing wastewater in a fixed-bed column system by surfactant-modified zeolite (smz). J Hazard Mater 166, 647-654.

Pan, B., Xing, B.S., 2008. Adsorption mechanisms of organic chemicals on carbon nanotubes. Environ Sci Technol 42, 9005-9013.

Park, S., Choi, K., 2008. Hazard assessment of commonly used agricultural antibiotics on aquatic ecosystems. Ecotoxicology 17, 526-538.

Patel, H., Vashi, R.T., 2012. Fixed bed column adsorption of acid yellow 17 dye onto tamarind seed powder. The Canadian Journal of Chemical Engineering 90, 180-185.

Sousa, F.W., Oliveira, A.G., Ribeiro, J.P., Rosa, M.F., Keukeleire, D., Nascimento, R.F., 2010. Green coconut shells applied as adsorbent for removal of toxic metal ions using fixed-bed column technology. J Environ Manage 91, 1634-1640.

Stafiej, A., Pyrzynska, K., 2007. Adsorption of heavy metal ions with carbon nanotubes. Sep Purif Technol 58, 49-52.

Ternes, T.A., Meisenheimer, M., McDowell, D., Sacher, F., Brauch, H.-J., HaistGulde, B., Preuss, G., Wilme, U., Zulei-Seibert, N., 2002. Removal of pharmaceuticals during drinking water treatment. Environ Sci Technol 36, 3855-3863.

Thiele-Bruhn, S., 2003. Pharmaceutical antibiotic compounds in soils - a review. J Plant Nutr Soil Sc 166, 145-167.

Tian, Y., Gao, B., Wang, Y., Morales, V.L., Carpena, R.M., Huang, Q., Yang, L., 2012a. Deposition and transport of functionalized carbon nanotubes in water-saturated sand columns. J Hazard Mater 213-214, 265-272. 
Tian, Y., Gao, B., Ziegler, K.J., 2011. High mobility of sdbs-dispersed singlewalled carbon nanotubes in saturated and unsaturated porous media. J Hazard Mater 186, 1766-1772.

Tian, Y., Morales, V., Wu, L., Wang, Y., Munoz-Carpena, M., Cao, C., Huang, Q., Yang, L., 2012b. Methods of using carbon nanotubes as filter media to remove aqueous heavy metals. Chem Eng J 210, 557-563.

Wang, X., Li, Q., Xie, J., Jin, Z., Wang, J., Li, Y., Jiang, K., Fan, S., 2009.

Fabrication of ultralong and electrically uniform single-walled carbon nanotubes on clean substrates. Nano Letters 9, 3137-3141.

Zhang, D., Pan, B., Zhang, H., Ning, P., Xing, B.S., 2010. Contribution of different sulfamethoxazole species to their overall adsorption on functionalized carbon nanotubes. Environ Sci Technol 44, 3806-3811.

Zhang, L., Petersen, E.J., Huang, Q., 2011. Phase distribution of (14)c-labeled multiwalled carbon nanotubes in aqueous systems containing model solids: Peat. Environ Sci Technol 45, 1356-1362. 


\section{Figure Captions}

Figure 1. Breakthrough curves of sulfamethoxazole (SMX) and sulfapyridine (SPY) in the CNT/sand fixed bed columns under various CNT incorporation methods. Constant conditions included: $\mathrm{pH}$ 5.6, $10 \mathrm{mg}$ CNTs, $15 \mathrm{~cm}$ bed depth, adsorbate initial concentration of $20 \mathrm{mg} / \mathrm{L}$, and $2 \mathrm{~mL} / \mathrm{min}$ flow rate.

Figure 2. Breakthrough curves of sulfamethoxazole (SMX) and sulfapyridine (SPY) in the CNT/sand fixed bed columns under various pHs. Constant conditions included: CNT incorporation by mixed method, $10 \mathrm{mg}$ CNTs, $15 \mathrm{~cm}$ bed depth, adsorbate initial concentration of $20 \mathrm{mg} / \mathrm{L}$, and $2 \mathrm{~mL} / \mathrm{min}$ flow rate.

Figure 3. Breakthrough curves of sulfamethoxazole (SMX) and sulfapyridine (SPY) in the CNT/sand fixed bed columns under various bed depths. Constant conditions included: CNT incorporation by mixed method, $\mathrm{pH} 5.6,10 \mathrm{mg}$ CNTs, adsorbate initial concentration of $20 \mathrm{mg} / \mathrm{L}$, and $2 \mathrm{~mL} / \mathrm{min}$ flow rate.

Figure 4. Bed Depth Service Time (BDST) model for (A) sulfamethoxazole (SMX) and (B) sulfapyridine (SPY) at breakpoint $\left(\mathrm{C} / \mathrm{C}_{0}=5 \%\right)$ and exhausted point $\left(\mathrm{C} / \mathrm{C}_{0}=95 \%\right)$. Constant conditions included: CNT incorporation by mixed method, $\mathrm{pH}$ 5.6, $10 \mathrm{mg}$ CNTs, adsorbate initial concentration of $20 \mathrm{mg} / \mathrm{L}$, and $2 \mathrm{~mL} / \mathrm{min}$ flow rate.

Figure 5. Relationship between changes in column capacity (mg/g) of (A) sulfamethoxazole (SMX) and (B) sulfapyridine (SPY) for 5 fixed-bed regeneration cycles. Sorption conditions were conducted consistently for mixed CNT incorporation, at pH 5.6, $10 \mathrm{mg} \mathrm{CNT}, 15 \mathrm{~cm}$ bed depth, adsorbate initial concentration of $20 \mathrm{mg} / \mathrm{L}$, and 2 $\mathrm{ml} / \mathrm{min}$ flow rate. 
Figure 1
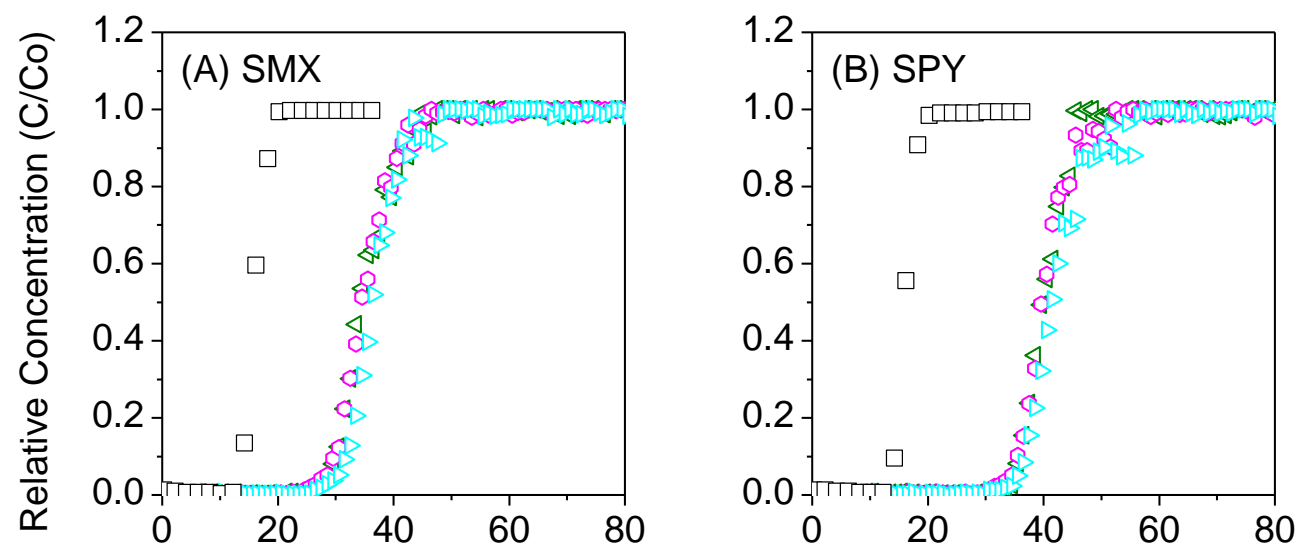

Duration (min)

Incorporation: $\square$ CNT-free $\triangleleft$ Layered CNT $\diamond$ Mixed CNT $\triangleright$ Deposited CNT

Figure 2
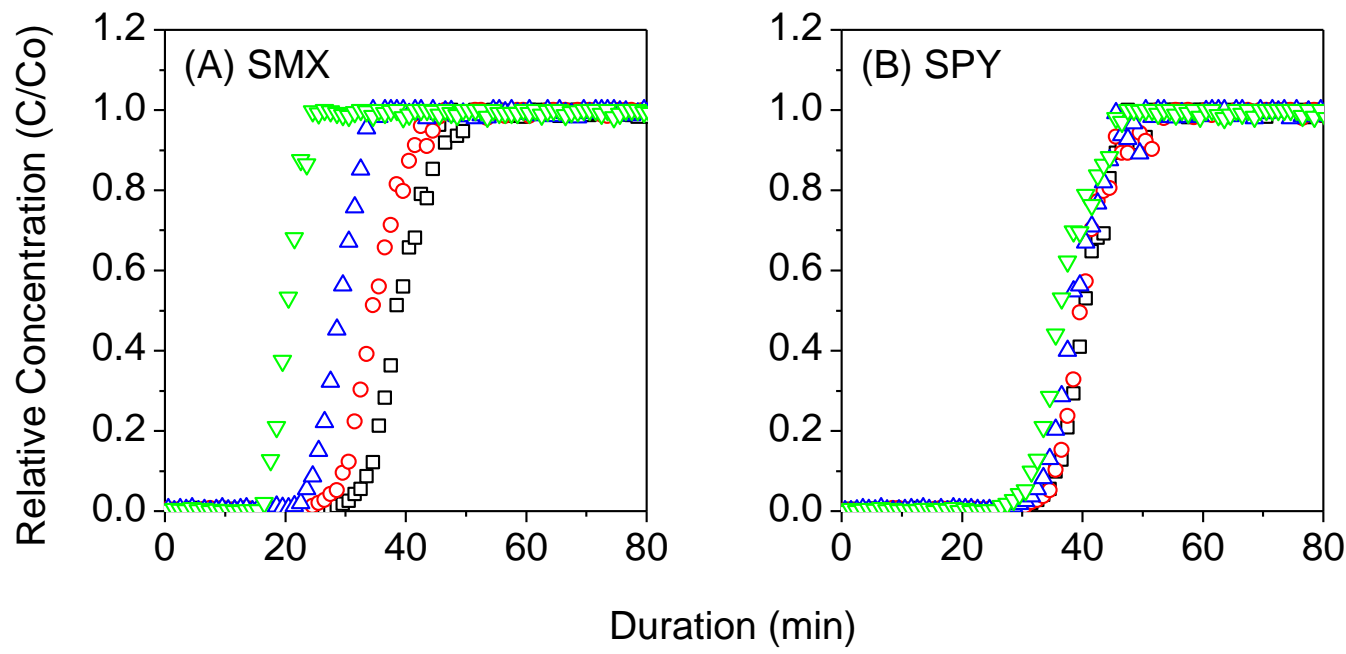

$\mathrm{pH}: \quad \square \quad 3.0 \quad 5.6 \quad 4.0 \quad \nabla .0$ 
Figure 3
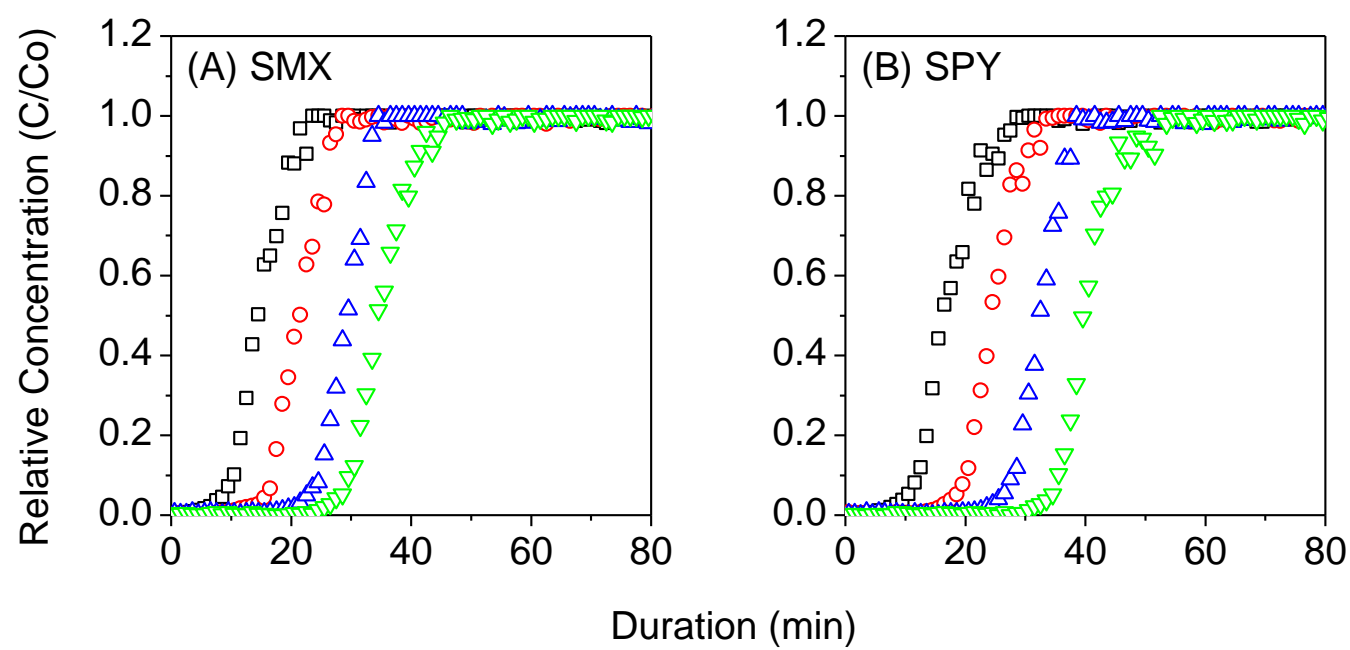

Bed depth: $\triangle 6 \mathrm{~cm} \circ 9 \mathrm{~cm} \triangle 12 \mathrm{~cm} \nabla 15 \mathrm{~cm}$

\section{Figure 4}
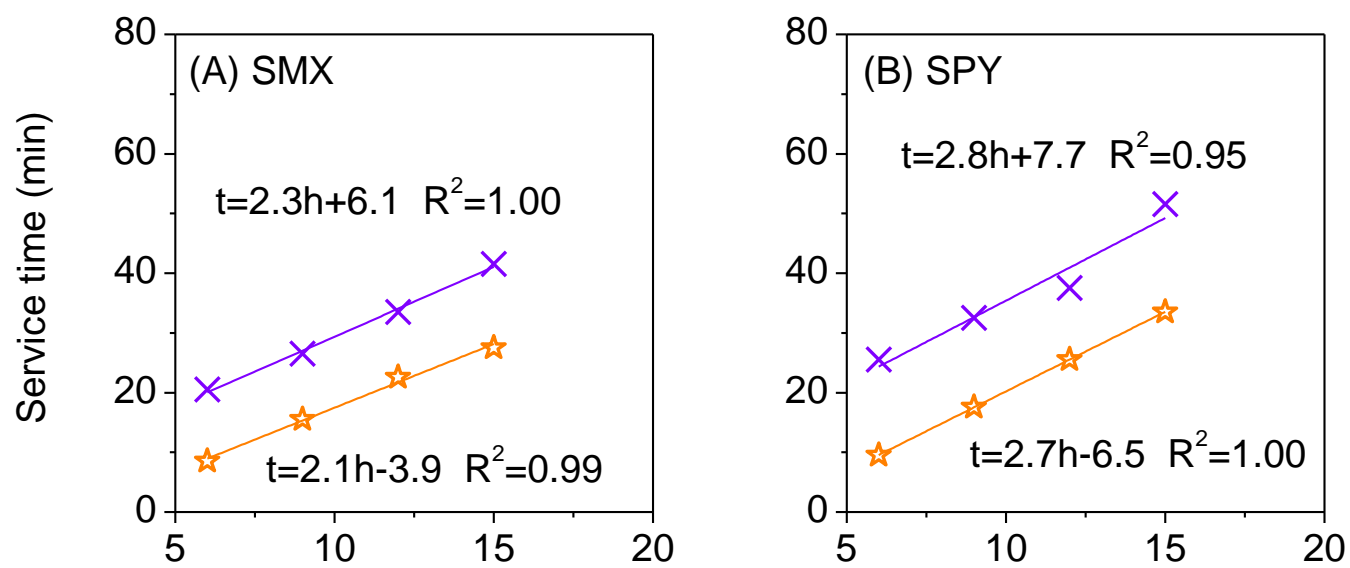

Bed depth $(\mathrm{cm})$

is Breakpoint $\times$ Exhausted point 
Figure 5
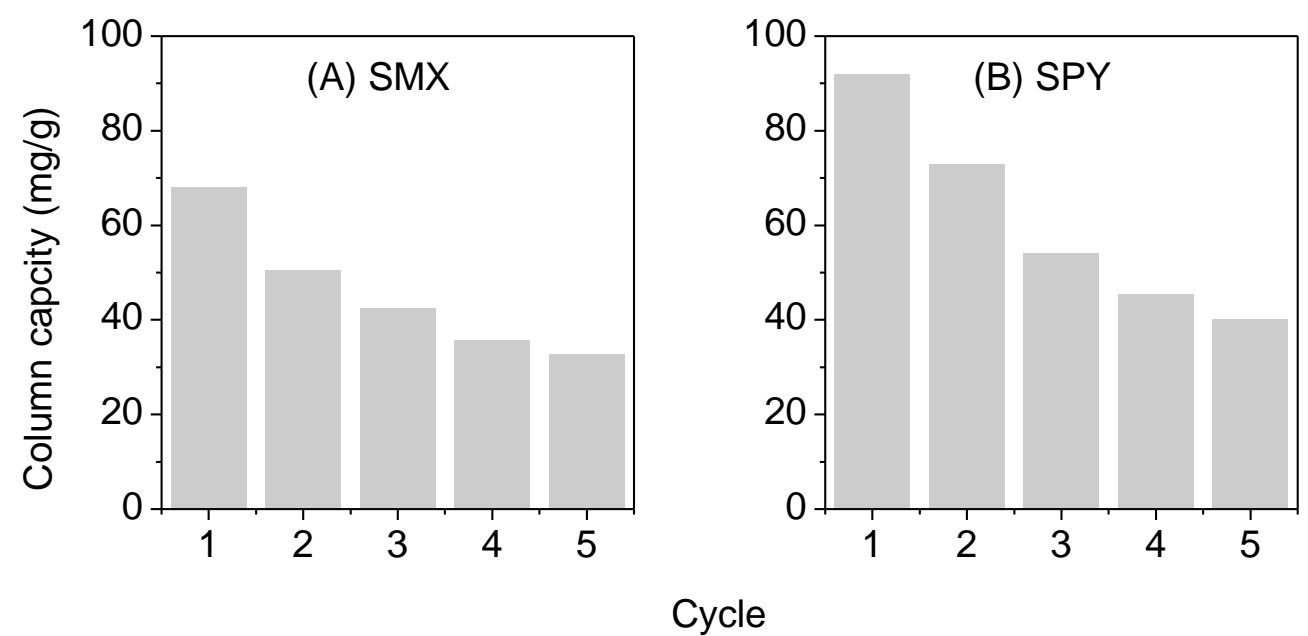
Tables

Table 1. Summary of transport and adsorption parameters of the two antibiotics in CNT/sand columns ${ }^{(1)}$.

\begin{tabular}{|c|c|c|c|c|c|c|c|c|}
\hline \multirow[b]{2}{*}{ Treatment } & \multicolumn{4}{|c|}{ SMX } & \multicolumn{4}{|c|}{ SPY } \\
\hline & $\begin{array}{c}\mathrm{t}_{\mathrm{b}} \\
(\mathrm{min})\end{array}$ & $\begin{array}{c}\mathrm{t}_{\mathrm{e}} \\
(\mathrm{min})\end{array}$ & $\begin{array}{c}\mathrm{v}_{\mathrm{z}} \\
(\mathrm{cm} / \mathrm{min})\end{array}$ & $\begin{array}{c}\text { Capacity } \\
(\mathrm{mg} / \mathrm{g})\end{array}$ & $\begin{array}{c}\mathrm{t}_{\mathrm{b}} \\
(\mathrm{min})\end{array}$ & $\begin{array}{c}\mathrm{t}_{\mathrm{e}} \\
(\mathrm{min})\end{array}$ & $\begin{array}{c}\mathrm{v}_{\mathrm{z}} \\
(\mathrm{cm} / \mathrm{min})\end{array}$ & $\begin{array}{c}\text { Capacity } \\
(\mathrm{mg} / \mathrm{g})\end{array}$ \\
\hline \multicolumn{9}{|l|}{ Incorporation } \\
\hline Layered & 28.5 & 43.5 & 0.42 & 68.5 & 34.5 & 44.5 & 0.37 & 89.4 \\
\hline Mixed & 27.7 & 44.5 & 0.43 & 69.3 & 33.6 & 51.5 & 0.37 & 91.9 \\
\hline Deposited & 29.6 & 47.5 & 0.41 & 77.7 & 35.4 & 55.4 & 0.35 & 97.8 \\
\hline \multicolumn{9}{|l|}{$\mathrm{pH}$} \\
\hline 3.0 & 31.7 & 49.7 & 0.38 & 84.9 & 33.4 & 50.4 & 0.36 & 93.9 \\
\hline 5.6 & 27.6 & 44.5 & 0.43 & 69.3 & 33.5 & 51.5 & 0.37 & 91.9 \\
\hline 7.0 & 22.6 & 32.7 & 0.53 & 45.8 & 31.6 & 49.3 & 0.39 & 86.1 \\
\hline 9.0 & 16.6 & 23.5 & 0.76 & 11.7 & 29.5 & 44.6 & 0.40 & 77.8 \\
\hline
\end{tabular}

$\underline{\text { Bed depth }}$

$\begin{array}{lrrrrrrrr}6 \mathrm{~cm} & 8.5 & 20.6 & 0.39 & 72.8 & 9.5 & 25.5 & 0.34 & 97.1 \\ 9 \mathrm{~cm} & 15.5 & 26.5 & 0.41 & 71.6 & 17.5 & 32.5 & 0.36 & 94.5 \\ 12 \mathrm{~cm} & 22.5 & 33.6 & 0.41 & 76.9 & 25.6 & 37.7 & 0.37 & 94.7 \\ 15 \mathrm{~cm} & 27.4 & 44.5 & 0.42 & 69.3 & 33.6 & 51.8 & 0.37 & 91.9\end{array}$

$\underline{\text { Adsorbent dosage }}$

\begin{tabular}{|c|c|c|c|c|c|c|c|c|}
\hline $10 \mathrm{mg}$ & 27.5 & 44.6 & 0.42 & 69.3 & 33.5 & 51.3 & 0.37 & 91.9 \\
\hline $20 \mathrm{mg}$ & 45.4 & 62.3 & 0.28 & 70.0 & 53.3 & 73.9 & 0.24 & 86.8 \\
\hline $30 \mathrm{mg}$ & 58.3 & 76.9 & 0.23 & 64.9 & 70.4 & 101.3 & 0.18 & 85.1 \\
\hline $40 \mathrm{mg}$ & 66.7 & 95.4 & 0.19 & 62.8 & 85.8 & 122.8 & 0.15 & 82.4 \\
\hline sorbate & & & & & & & & \\
\hline $10 \mathrm{mg} / \mathrm{L}$ & 37.4 & 53.5 & 0.33 & 54.8 & 51.8 & 62.4 & 0.26 & 80.9 \\
\hline
\end{tabular}




$\begin{array}{lllllllll}20 \mathrm{mg} / \mathrm{L} & 27.4 & 44.5 & 0.42 & 69.3 & 33.7 & 51.4 & 0.37 & 91.9 \\ 30 \mathrm{mg} / \mathrm{L} & 26.6 & 37.5 & 0.48 & 84.8 & 25.5 & 46.8 & 0.42 & 109.6 \\ 40 \mathrm{mg} / \mathrm{L} & 25.6 & 32.5 & 0.52 & 92.0 & 25.5 & 41.5 & 0.45 & 123.4\end{array}$

Flow rate

$\begin{array}{lcccccccc}1 \mathrm{ml} / \mathrm{min} & 59.3 & 93.5 & 0.20 & 78.3 & 67.0 & 103.2 & 0.18 & 94.9 \\ 2 \mathrm{ml} / \mathrm{min} & 27.7 & 44.7 & 0.43 & 69.3 & 33.6 & 51.3 & 0.37 & 91.9 \\ 3 \mathrm{ml} / \mathrm{min} & 19.8 & 27.7 & 0.65 & 67.7 & 22.3 & 29.7 & 0.55 & 90.2 \\ 4 \mathrm{ml} / \mathrm{min} & 14.8 & 19.9 & 0.91 & 61.9 & 16.8 & 21.7 & 0.79 & 83.6\end{array}$

(1) The parameters include breakpoint time $\left(t_{b}\right)$, exhausted time $\left(t_{e}\right)$, adsorption zone velocity $\left(\mathrm{v}_{\mathrm{z}}\right)$, and column capacity. 
Table 2. Definition and formula of the primary adsorption zone (PAZ) parameters used in this study.

\begin{tabular}{|c|c|c|c|}
\hline Parameter & Unit & Definition & Formula \\
\hline$h$ & $\mathrm{~cm}$ & Bed depth & \\
\hline$C_{0}$ & $\mathrm{mg} / \mathrm{L}$ & Influent antibiotic concentration & \\
\hline$C_{t}$ & $\mathrm{mg} / \mathrm{L}$ & $\begin{array}{l}\text { effluent antibiotic concentration at time } t \text { during the } \\
\text { experiment }\end{array}$ & \\
\hline$t_{b}$ & $\min$ & $\begin{array}{l}\text { Breakpoint time when the normalized effluent } \\
\text { concentration }\left(\mathrm{C} / \mathrm{C}_{0}\right) \text { reaches } 5 \%\end{array}$ & \\
\hline$t_{e}$ & $\min$ & $\begin{array}{l}\text { Time when } \mathrm{C} / \mathrm{C}_{0} \text { reaches } 95 \% \text {, also defined as the } \\
\text { time when the column is exhausted/spent }\end{array}$ & \\
\hline$t_{z}$ & $\min$ & $\begin{array}{l}\text { Time required for the PAZ to move through the fixed- } \\
\text { bed column after it has become established }\end{array}$ & $t_{z}=t_{e}-t_{b}$ \\
\hline$F$ & - & $\begin{array}{l}\text { Fraction of the PAZ that still capable of adsorption } \\
\text { (i.e., unspent adsorbent fraction in the PAZ) }\end{array}$ & $F=\frac{\int_{t_{b}}^{t_{e}}\left(1-\frac{C_{t}}{C_{0}}\right) d t}{t_{e}-t_{b}}$ \\
\hline$t_{f}$ & $\min$ & $\begin{array}{l}\text { Elapsed time between initial injection and the } \\
\text { breakpoint, also defined as the time required for the } \\
\text { initial formation of the PAZ }\end{array}$ & $t_{f}=(1-F) t_{z}$ \\
\hline$v_{z}$ & $\mathrm{~cm} / \mathrm{min}$ & Velocity of PAZ progressing through the bed & $v_{z}=\frac{h}{t_{e}-t_{f}}$ \\
\hline$h_{z}$ & $\mathrm{~cm}$ & Height of the adsorption zone & $h_{z}=v_{z} t_{z}$ \\
\hline$s$ & - & $\begin{array}{l}\text { Saturation percentage of the total column at } \\
\text { breakthrough }\end{array}$ & $s=\frac{h+(F-1) h_{z}}{h} \times 100$ \\
\hline
\end{tabular}

